\title{
Panoramic UAV Views for Landscape Heritage Analysis Integrated with Historical Maps Atlases
}

\author{
Raffaella Brumana, Daniela Oreni, Mario Alba, Luigi Barazzetti, \\ Branka Cuca, and Marco Scaioni \\ Politecnico di Milano, piazza Leonardo Da Vinci 32, Milan, Italy \\ raffaella.brumana@polimi.it
}

\begin{abstract}
Analysis of landscape heritage and territorial transformations dedicated to its protection and preservation rely increasingly upon the contribution of integrated disciplines. In 2000 the European Landscape Convention established the necessity 'to integrate landscape into its regional and town planning policies and in its cultural, environmental, agricultural, social and economic policies'. Such articulated territorial dimension requires an approach able to consider multi-dimensional data and information from different spatial and temporal series, supporting territorial analysis and spatial planning under different points of view. Most of landscape representation instruments are based on 3D models based on top-down image/views, with still weak possibilities to reproduce views similar to the human eye or map surface development along preferential directions (e.g. water front views). A methodological approach of rediscovering the long tradition of historical water front view maps, itinerary maps and human eye maps perspective, could improve content decoding of cultural heritage with environmental dimension and its knowledge transfer to planners and citizens. The research here described experiments multiple view models which can simulate real scenarios at the height of observer or along view front. The paper investigates the possibilities of panoramic views simulation and reconstruction from images acquired by $R C / U A V$ platforms and multisensory systems, testing orthoimage generation for landscape riparian areas and water front wiew representation, verifying the application of automatic algorithms for image orientation and DTM extraction (AtiPE, ATE) on such complex image models, identifying critical aspects for future development. The sample landscape portion along ancient water corridor, with stratified values of anthropogenic environment, shows the potentials of future achievement in supporting sustainable planning through technical water front view map and $3 D$ panoramic views, for Environmental Impact Assessment (EIA) purposes and for the improvement of an acknowledged tourism within geo-atlas based on multi-dimensional and multitemporal Spatial Data Infrastructures (SDI).
\end{abstract}

Keywords: landscape heritage, UAV, image orientation, panoramic views, historical maps

\section{Introduction}

The paper relates on-going experiments driven by the necessity to provide new scenarios for retrieving geospatial knowledge of territory and instruments capable of managing informa- 
tion to better addresses landscape heritage policies. In this sense, the advanced geo-web instruments can give a contribution to support the preservation of ancient traces of precious anthropogenic environment, partly recognizable within the context and to provide a better comprehension of the participated citizen culture of territory.

Experiences carried out within the Atl@nte geoportal (an on-line open-source atlas of historical cadasters and topographic maps of Lombardy, www.atlantestoricolombardia.it), have provided a valid ground to compare the current landscape with different historical layers. Furthermore, they ask to enhance the comprehension of such complex areas introducing innovative representation and rediscovering the semantic content potential of ancient views for anthropogenic landscape interpretation and identity recognizing process by the people, unavoidable elements during the preservation process. A significant and well representative case study in the Italian Alpine foothills (pre-Alpine region) is reported and discussed. The possibility of utilising image sequences gathered by Unmanned Aerial Vehicles (UAV) combined with existing Digital Terrain Models (DTM) was investigated in order to obtain 3D textured models for landscape analysis, especially in areas featuring strong vertical edges (built environment of hills, coast, mountains, and the like). A model helicopter, flying at few tens of metres from the ground, has been tested to acquire images all around a given point. The composition of the images to form panoramic views can be used to reproduce the 'human' point of view at a certain location. This kind of landscapes cannot be represented in a realistic way using airborne imagery which are normally applied for state-of-the-art landscape 3D modelling.

According to landscape policies in EU and Italian frameworks, the necessity to develop new tools for representation to be used for landscape planning simulation (Sect. 1) has been discussed. These include experimentation of RC/UAV images for panoramic views reconstruction (Sect. 2). A case study area has been selected to support ancient view simulation for scenario analysis and touristic valorisation of historic itinerary road (Sect. 3). The problem of image-orientation for non-conventional image acquisition dedicated to orthoimage projection of non-conventional views has been discussed relating to sensor system RC/UAV platform and GPS digital camera (Sect. 4). The promising potentials of this approach for the future strengthening of e-contents through web atlas and modern devices are illustrated in Section 5.

\subsection{EU and Italian legislative framework for landscape planning and sce- narios simulation}

The Italian legislative framework on the protection of the landscape has been developed according to the European Landscape Convention [16], stipulated in Florence in 2000 and ratified by the Italian government in January 2006. Here the landscape of every country includes the historical, monumental and the natural characteristics of the territory, considered as part of the cultural heritage of all European citizens. Identity and recognition become two key features of the landscape quality and contribute to the formation and the increasing of the individual and social quality of life. The landscape thus becomes a resource for sustainable development of all countries, with the result that the whole territory must be considered in the plans and programs to enhance the landscape, with the attention directed not only to the 'exceptional' places, but also to the 'everyday life landscapes and degraded landscapes'.

Acting on the recommendations contained in the European Spatial Development Perspective 
(ESDP), prepared by the EU in May 1999 in Potsdam, the EU Landscape Convention also imposes the need for each Country to integrate landscape into regional and town planning policies, in cultural, environmental, agricultural, social and economic policies, as well as in any other policies with possible direct or indirect impact on landscape. The objective is to formulate general application principles, strategies and guidelines that permit the taking of specific measures aimed at the protection, management and planning of landscapes. In this frame, the Code of cultural heritage and landscape (DL2 N.42/2004, [18]) stated the procedures for the landscape granting, assigning the tasks of monitoring projects to the Architectural Heritage and Landscape Office, the Italian Ministerial body in charge of protection, called upon to make an assessment on the project proposals. Its opinion has a binding force for the Regions, the agency delegated for the final authorisation, which in turn must ensure that the projects will respect the regulatory guidelines for provincial and municipal planning, as contained in the Regional Landscape Territorial Plan. The legislative objective, through this whole process of approval, is to lead transformation of landscape taking into account the morphology of the places, the scenic and environmental context, and the traces of their history, and not to overlap uncritically and brutally to existing landscape. The Code made compulsory the submission of the 'report on landscape' together with the application for authorization, both essential for the assessment, made by the competent authority, of the project in relation to the elements of landscape value, highlighting the impact of the projects on the landscape and the elements of mitigation and compensation required. Furthermore, landscapes along the water paths and coastal areas are considered as regions of a great strategic importance for the EU hence in this sense, INSPIRE directive [18] together with Shared Environment Information System (SEIS) are identified as main tools to facilitate the information flow in these areas.

Regarding the assessment of the landscape compatibility of the transformation proposed, it is prescribed to submit synthetic analyses of both state-of-the-art and the project. They must include not only descriptions, current or historical map extracts, but especially detailed simulations, made by 'realistic photo-modelling that includes an appropriate area around the project, calculated from the ratio of existing intervisibility'. The 'photographic representation' of the project area and of the landscape contest must be taken from accessible places and/or scenic routes, as specified by the Scottish Natural Heritage for landscape management Environmental Impact Assessment (EIA) [17]. It must include fronts, skylines and visual perspective from which the transformation is visible, with particular reference to high visibility areas (e.g. slopes, coasts). In this context, the development of innovative 3D metric representation of the landscape is an improvement to the traditional 2D photographic representations of panoramic views.

\section{Sensors for Landscape Image Processing: State of Art}

Development of RC/UAV sensors for documentation, inspection and surveying of Cultural Heritage is a field partially explored with success in Archeology [5], by inheriting the horizontal flight of aerial photogrammetry and image orientation.With reference to Landscape and Environmental Heritage domain, different problems have to be investigated in photogrammetric $\mathrm{RC} / \mathrm{UAV}$ applications, regarding image acquisition and orientation for 3D reconstruction of landscape with a complex morphology, needing unconventional camera poses, vertical images or/and oblique images. The need of an accurate flight planning and control requires data acquisition to be carried out along a regular path. Indeed, photogrammetric surveys 


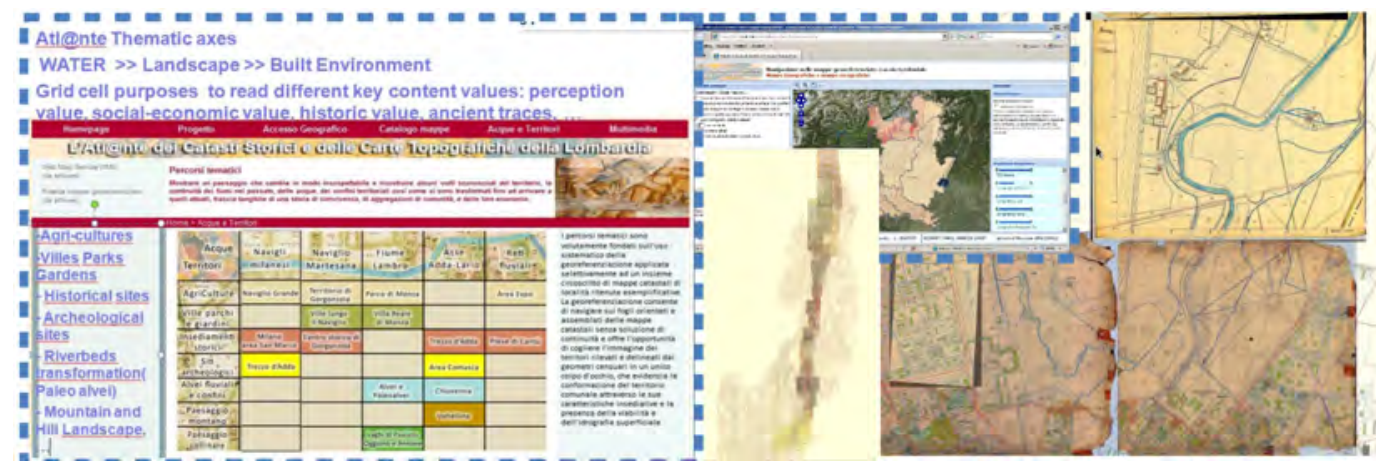

Figure 1: Atl@s web geoportal and the landscape area test along thematic axe of the Lambro river

need a block of images taken from different points of view at suitable scales and baselines. The integration with GNSS/INS $[3,4,6]$ sensors allowed to obtain satisfying results, at the landscape scale. Camera calibration and image orientation have reached a high level in term of automation [8]. In the case nadiral images, the use uncontrolled flight path under the condition of well-targeted ground control points (GCP) enable the application of algorithms for automatic orientation. Research on fully automatic UAV image-based sensor orientation [8] opens opportunities in contexts like water view fronts devoted to metric image processing and information extraction.Moving the point of view, the GCP detection for the complex schema image block become difficult, especially in the case of very low cost uncontrolled flight, that ask to develop orientation and 3D modelling from markerless images [11] considering the wide spread potential of similar applications. Controlled sensor systems could be matched with the results obtained by multiple views 3D texturized models in the Computer Vision domain (Acute3D, http://acute3d.com/) in order to obtain technical instrument for line-of-sight analysis.

\section{SDI Integration with Ancient View Simulation for Scenario Reconstruc- tion and Touristic Valorisation of Historic Itineraries}

Landscape heritage analysis, safeguarding and preserving can benefit from the integration of multi spatial-temporal data within a Spatial Data Infrastructure (SDI), rediscovering ancient map views of territory and different point of views of landscape observation [14]. The Atl@nte geoportal has provided a valid ground to deeper investigate and compare the current landscape with different stratified historical layers (Fig. 1) through the WMS/WFS generated on georeferenced ancient cadastral maps related to the current map (Google ${ }^{\circledR}$ imagery, 3D orthophoto maps, such as Terraitaly ${ }^{\circledR}$, large scale technical map, etc.). Such a geo-metric grid allows to superimpose different chronological series, extracting information about transformations, permanences and mutations, especially if strictly related to the in situ data collection on the stratigraphic units, based on chrono-typological and archeometric approach [2].

A study case along water axes within Atl@nte was selected in the Italian pre-Alpine region. Motivation of this choice were the rich historic background and the complex landscape morphology of natural and built environment. Both aspects showed the potentials of providing new scenarios for retrieving geospatial knowledge of territory and instruments capable to sup- 
port landscape heritage knowledge, safeguarding policies, addressing planning and divulgation instruments among citizens for sustainable tourism.

Rediscovering the perception of landscape value and synthesis represented in the past through observer point of views, generating 'vedute' and perspective maps, water front views of portion of landscape faced on the inland water paths or lakes, that can be very useful if gradually integrated within modern geo-portal, introducing an added value of territorial map representation.

Architectural survey is usually obliged to move the point of view respect different plans of interest in function of the different structural elements (vertical fronts, facades, horizontal planes, floors, vaults). On the other hand, landscape representation is progressively trying to overcome the unique traditional nadiral privileged point of view represented by the aerial images acquired for current 2.5D cartography. Different point of views have to be gradually incorporated, as in the case of 3D city model navigation (e.g. Google Earth ${ }^{\circledR}$ ) obtained by the individual projection of rectified images on building facades. The experimentation of mobile sensor platform image acquisition using an $\mathrm{RC}$ helicopter to reconstruct has been planned to reconstruct ancient panoramic views that represent an important witness of the great value assigned to this portion of landscape. The manifold aspects of interest will be addressed to in Section 1.2. In the conviction that rediscovery of ancient views can contribute to the rediscovery of the stratified values to preserve this area within planning policies and to valorise divulgation of touristic circuits, helping perception by the people using geo-portal, smart phone devices to strengthening comprehension of the historic geography of such places. In particular, a test was carried out on a simulation of ancient views generally acquired from privileged point of view (such as bell towers) on 'strategic' zones of territory, as in the case of 'non-rigorous' or quasi-perspective maps, such as the 'Pastoral Visits to the Pievi' by San Carlo Borromeo in Lombardy, XVI century (Fig. 3). Panoramic image acquisition by $\mathrm{RC} / \mathrm{UAV}$ flight simulation has been experimented in order to verify the potentials and critical aspects for innovative representations of stratified multi-spatial/multi-temporal landscape reconstruction of complex strategic areas developed in the centuries along the ancient great itinerary roads. The sample test-site represents an important node along the Roman road from Aquileia to the North Europe, developed upon Neolithic settlement discovered around the area for favourable climate condition, water resources. This area featured a rich anthropogenic stratified territory with a natural defensive morphology. Celtic, Roman and Lombard archaeological settlement were found in the 'Park of Barro', and with the important Lombard-Benedictine Monastery of San Pietro al Monte, Civate. Despite the richness of this area we miss a synthetic view at $360^{\text {circ }}$ able to transfer those values to avoid the destroying intervention recently occurred by the new tunnel access exactly in correspondence of this node. An alternative solution, not completely far from the adopted one, would have more safeguarded this place.

A possible contribution to the divulgation of a participated citizen culture, by preserving ancient traces of precious landscape values conserved till now, can be given by advanced geo-web instruments.

Atl@s web can support historic geography and stratified ancient traces here represented on the traditional DTM and satellite imagery. The immediate perception given by the geospatial representation can be improved by privileged point of view of interest that can be usefully integrated to enhance the comprehension of beautiful natural and anthropogenic context 


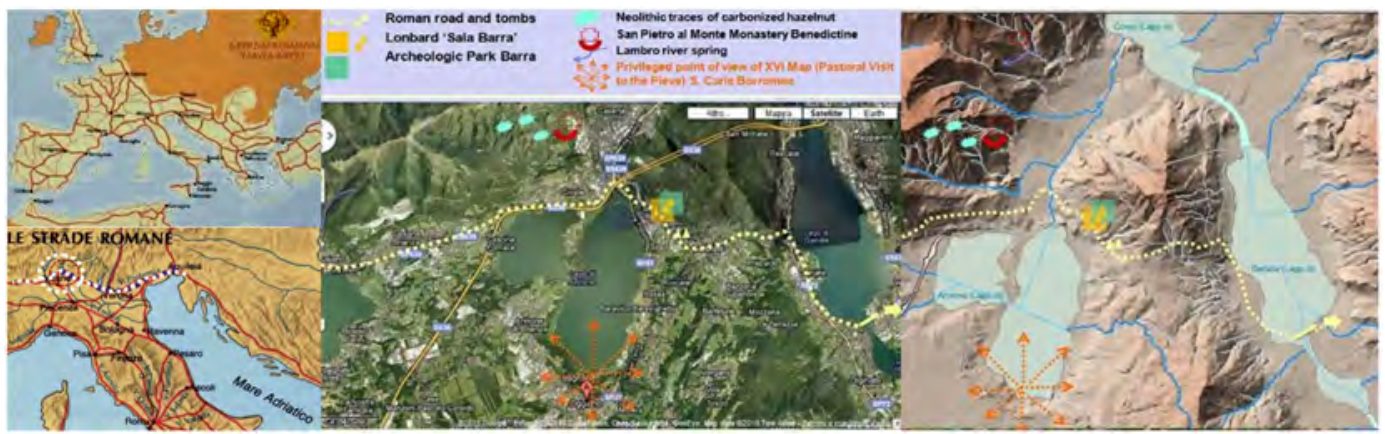

Figure 2: The sample landscape area, on the Lake of Annone closed to the access to the Lake of Como, is placed along an important node of the roman road from Aquileia to the North Europe. The principal stakeholder stratified landscape element are signalized.

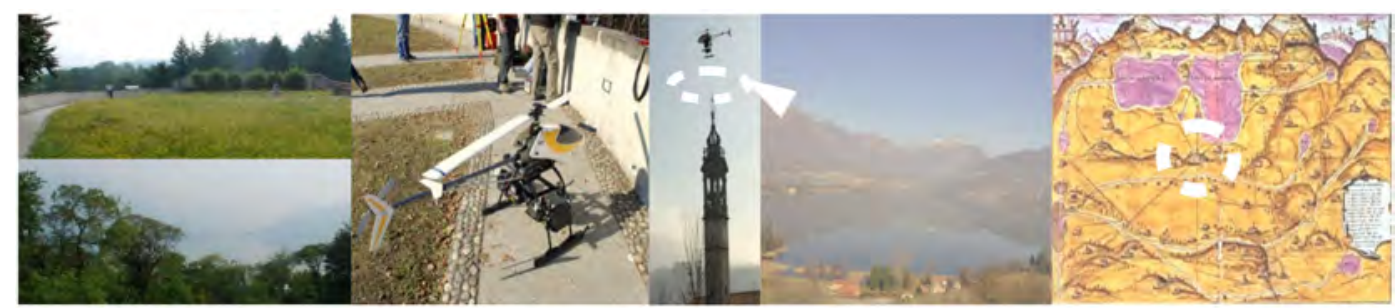

Figure 3: Images acquired from ground level (left) do not provide an overall view of the ancient map (right). Panoramic images (centre) acquired at the height of the bell tower using RC platform flight, allowed to reconstruct the privileged point of view of XVI century Map of the Pastoral Visit to the Pieve by S. Carlo Borromeo acquired from the Bell Tower of the Church s. Eufemia and Romanic Baptistery.

unknown by the people at the local municipality and global level.

\section{Non Controlled Flight and Controlled Sensor System for Landscape Or- thoimages Generation from Panoramic Views}

One of the aims of this project is the creation of panoramic images in order to texturize different landscapes. Since the approach is based on images taken from the ground instead of imagery used for aerial photogrammetric applications, some occlusions can be present. They can degrade the quality of the final results. This means that several objects located between the camera position and the investigated elements can be mapped generating a false result. On the other hand, this problem could be (partially) solved by employing images taken from different positions, with an interactive selection of the portions to be used.

Images acquired by an RC model helicopter have been used for high resolution modelling of the ground, tested on a portion of terrain with measured GCPs, while images acquired by digital camera integrated with GPS allows to solve orientation problem in order to obtain a $3 \mathrm{D}$ ortho-projection of a water view front portion without GCPs. 


\subsection{Flight test and Panoramic UAV images: data processing critical aspect}

Automatic image orientation by AtiPE and automatic high resolution DTM extraction by using PMVS2 or LPS eATE techniques are here discussed with reference to the sensor system equipped on the platform and to the context. Furthermore, UAV images have been also used for high resolution modelling of the ground, tested on a portion of terrain with measured GCPs. In this experience, an automatic processing pipeline for the production of high resolution orthoimages has been setup. This includes automatic image orientation by AtiPE [8] and automatic high resolution DTM extraction by using PMVS2 or LPS eATE techniques. The former can be used to compute the exterior orientation (EO) through aerial triangulation of images taken with a calibrated camera, without any user interaction. In order to produce high resolution orthoimages or textured models from airborne sensors, terrain models derived from oriented UAV images using state-of-the-art matching techniques will be further investigated. Eventually, different software packages for orthoimage production from UAV data have been tested and evaluated according to image configuration and resolution. To check the metric accuracy achievable with the UAV system a photogrammetric block made up of 25 images was acquired in the area test over the municipality of Oggiono (Lombardy, Italy) on the top of a small hill with a dominant horizontal extension (Fig. 4). The UAV platform gives the opportunity to mount different types of cameras, according to the aim of each specific survey. In this case, a calibrated Nikon D90 (4288x2848 px) equipped with a $20 \mathrm{~mm}$ Sigma lens was employed. This camera has also the capability of acquiring video sequences. The network geometry includes images with a high overlap. Some of these were also quite oblique in order to obtain a better reconstruction of the elements over the horizontal plane (e.g. walls, columns, statues). The images were oriented with the ATiPE procedure [11], using SIFT features automatically extracted for several combinations of image pairs. To obtain a georeferenced result, 20 targets were placed on the ground. They were measured with a combined theodolite/GPS survey. 9 points were employed as GCPs in the bundle adjustment, while the remaining ones were used to check the quality of the estimated EO parameters. The coordinates of the centres of all targets were measured with the LSM algorithm, obtaining very precise images coordinates. The average height the above ground during the flight was ca $14 \mathrm{~m}$. RMSE values found were $5 \mathrm{~mm}$ in the horizontal plane $(x-y)$ and $17 \mathrm{~mm}$ along the vertical direction $(z)$.

Figure 5 shows a panoramic image generated by combining different pinhole shots. The object to be mapped is on the other side of the lake, facing the Roman road, the Benedictin Monastery and the Park of Barro. However, several other images were collected to obtain a final panorama with a large field of view, imaging also object in the nearby. A global visualization of the image can give nice results (although the weather conditions was not optimal due to mist). However, the final image is quite clean in correspondence of the target object, while for closer elements (e.g. trees, bell tower) some parallax errors were found.

\subsection{Panoramic image for 3D Spatial Database texturing}

The availability of an accurate 3D Spatial Database of the region allows to obtain a model usable for landscape representation purposes. This approach overcomes DTM extraction in order to generate panoramic photo projection of view front images acquired along riparian areas. Landscape visualization and photomontage experiments in the field of line-of-sight 


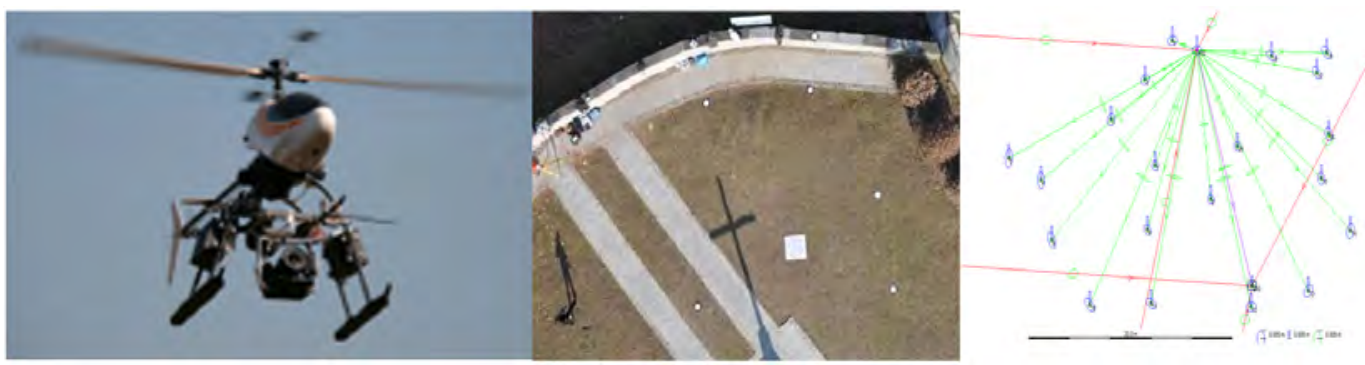

Figure 4: The UAV system, an image of the area and the scheme of theodolite/GPS measurements.

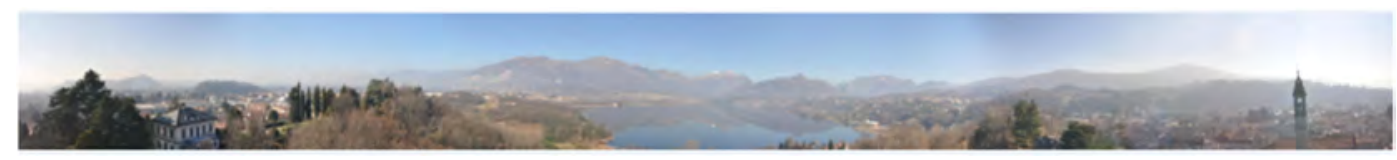

Figure 5: The panoramic image generated from a model helicopter from the ancient view point.

map integration are beginning to implement visual impact assessment tools [12] in order to enhance metric approach with respect to qualitative approach and passive auto stitching software packages [1]. Advanced algorithms developed for textured 3D models on panoramic images acquired by controlled sensor have been utilised. Integration of GPS sensors with digital camera has simulated fully equipped flight control experimented in case of close range acquisition by ground instead of by flight; implementation of RC platform with this sensor system would allow to enhance the orientation of such geometry for orthoprojection purposes. Here is described a methodology for automatically texture a 3D Spatial Database by oriented panoramic image and the results of the test application carried out on the shore of Lake Como, at the appendix node of the area test. In order to obtain a more complete acquisition of the lake-front different images have been acquired for each standpoints (Fig. 6). Photogrammetric surveys was carried out with a full-format Nikon D700 (4256x2832 pixels) camera with calibrated $90 \mathrm{~mm}$ lens. Each image was georeferenced with the so called 'photo-GPS' technique [9], where RTK-GPS measures of the camera positions and image are acquired together. For each position a set of images was acquired with 'photo-GPS' system, maintaining the same coordinates of image location while the orientation angles changed. Three panoramic images were generated from the images acquired at different stand points (Fig. 7).

The tie points of panoramic images were automatically extracted. Finally the camera positions and photogrammetric observations are adjusted together in order to obtain the EO parameters in WGS84 reference system. The oriented panoramic image has been automatically projected on the 3D Spatial Database (1:2,000 scale) and the first results are shown in Figure 8.

\section{Conclusions}

With reference to the sensor system, the implementation of controlled flight equipped on the platform with camera GPS allows to support panoramic image processing using automated 


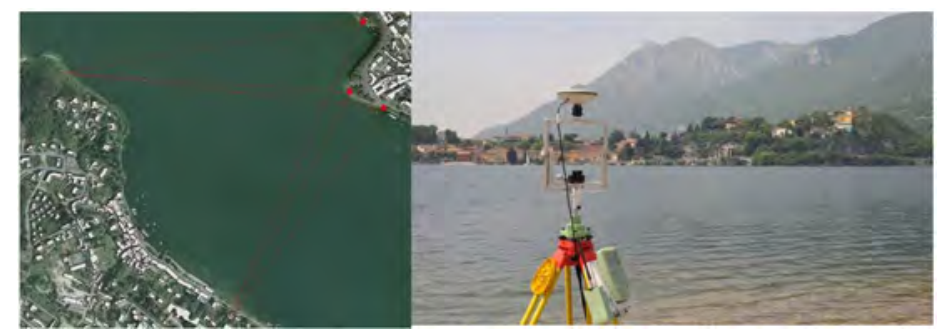

Figure 6: (Left) Test site, in red different standpoints and the lines-of-sight. (Right) 'PhotoGPS' system during the acquisition of images.

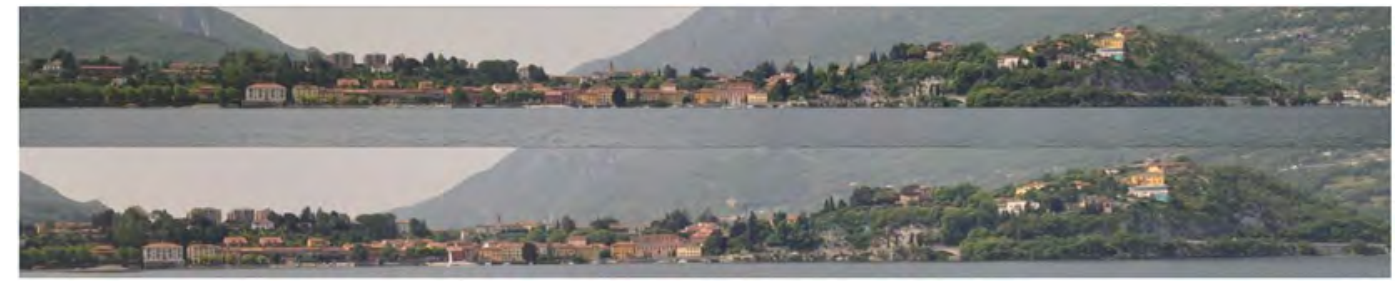

Figure 7: Two of the three Panoramic image acquired from different stand-points

techniques for image orientation and 3D surface reconstruction. Many critical aspects of the panoramic image projection of water coast views to obtain metric water front has to be faced and deeper investigated: segmentation of orthoimage projection on a 3D Spatial Database with the projection of portions of panoramic views along the sloped coast areas, analysis of geometric congruence and condition to synchronize the exact projection of the built up portion located on the second and third plane respect the foreground acquired on the close up building faced on the coast line. The research will continue the tests on these products, to support metric textured model generation and management within easy access software environment used by planners and professionals in order to generate a widely useful technical instrument. Developing multiple-choice representation would straighten the comprehension of complex scenarios, such as historic road and ecosystem corridors rediscovering new interpretation of the ancient itinerary maps and bird eye perspective contents. Modern devices integration to web geo-portal (such as smart phone, Ipad applications) could allow an on-site touristic divulgation and data sharing with local growth of sustainable touristic itineraries and identity process of knowledge and consciousness by the citizen. The sample area on landscape portion along water corridor with multiple stratified values of anthropogenic environment has shown the potentials of future achievements in this research field through a complementary integration of innovative technical map within atlas based on multi-temporal SDI: they contribute to enhance the comprehension of complex landscape portions, rediscovering the semantic content of ancient views for anthropic landscape analysis and identity recognizing process by the people, an unavoidable component in the preservation process.

\section{References}

[1] M.Brown, D.G. Lowe, 2005. Automatic Panoramic Image Stitching using Invariant Features, Int. Journal of Computer Vision, Volume 74, Number 1, 59-73, DOI: $10.1007 / \mathrm{s} 11263-006-0002-3$.

Geoinformatics FCE CTU 9, 2012 

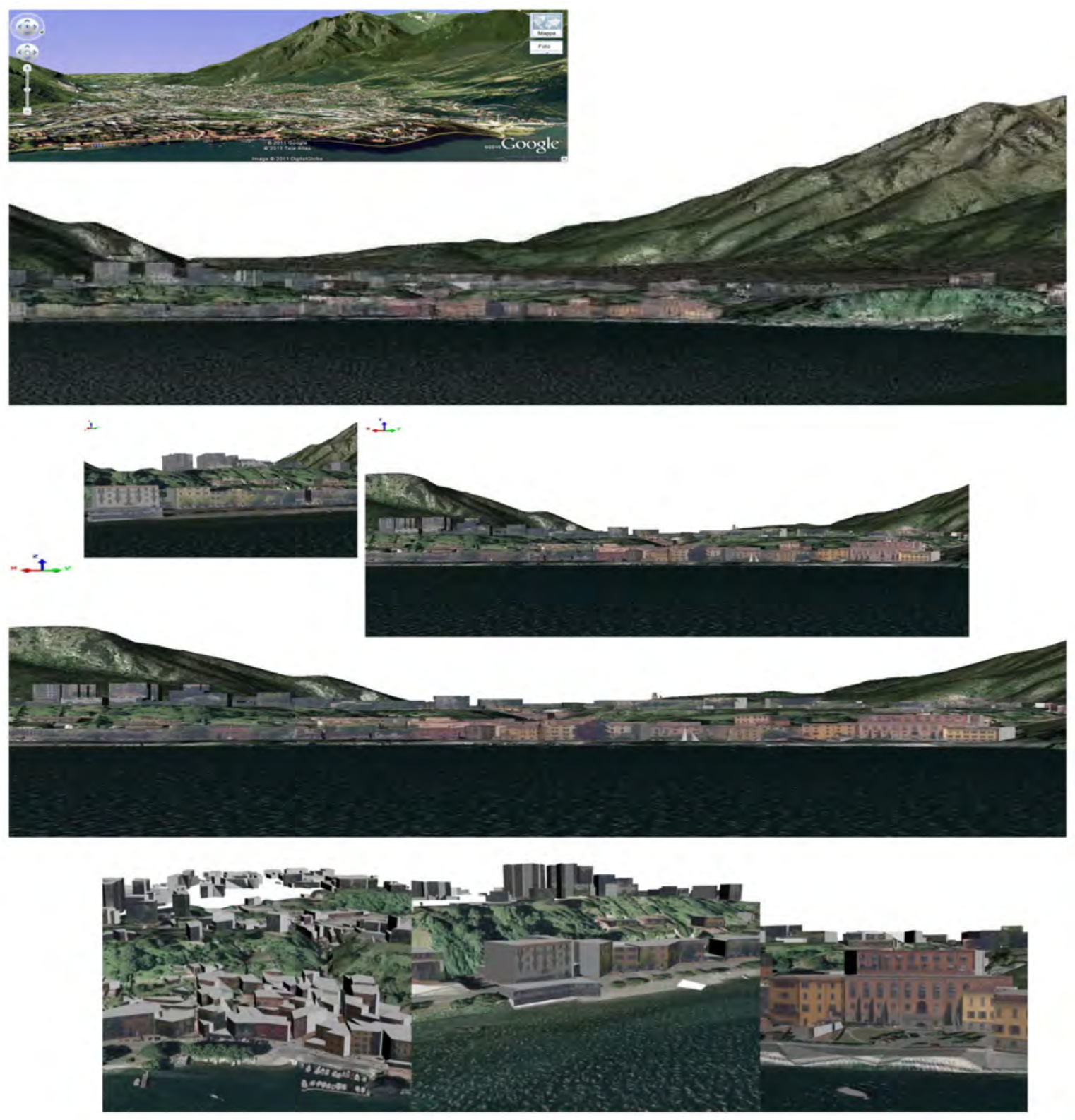

Figure 8: Figure. 8 (Above) Images of TopographicDB automatically textured by oriented panoramic images. (Below) VRLM model render images done within modelling sw environment (3DStudioMax)

[2] Brumana, R., Achille, C., Prandi, F., Oreni, D., (2006). 3D Data model for representing an historical Centre Site UDMS'06 25th Urban Data Management Symposium UDMS Aalborg, Denmark 1-8 9 Part IV

[3] Colomina, I. et al, 2007. The uVISION project for helicopter-UAV photogrammetry and remote-sensing. 7th Geomatic Week, Spain.

[4] Wang, J., 2008. Integration of GPS/INS/Vision Sensors to Navigate Unmanned Aerial 
Vehicles. IAPRSSIS, 37(B1): 963-9.

[5] Bendea, H.F., Chiabrando, et altri, 2007. Mapping of archaeological areas using a lowcost UAV the Augusta Bagiennorum Test site. In Proc. XXI Int. CIPA Symp, Athens, Greece, on CDROM.

[6] Bento, M.D.F., 2008. Unmanned Aerial Vehicles: an Overview. InsideGNSS (Jan/Feb 2008): 54-61. [7] Eugster, H., S. Nebiker, 2008. UAV-Based Augmented Monitoring - Real-Time Georeferencing and Integration of Video Imagery with Virtual Globes. IAPRSSIS, 37(B1): 1229-1235

[7] Barazzetti L., Remondino F., Scaioni M., Brumana R., 2010. Fully Automatic UAV Image-Based Sensor Orientation. In Proc. of ISPRS Comm. I Symp., Calgary (Canada), IAPRSSIS 38(1), on CDROM, 6 pp.

[8] Forlani, G., Pinto, L., 2007. GPS-assisted adjustment of terrestrial blocks. In: Proc. of the 5th Int. Symp. on Mobile Mapping Technology (MMT'07). Padova, ISSN 1682-1777, CD-ROM, pp1-7.

[9] Wang, M., Bai, H., Hu, F., 2008. Automatic Texture Acquisition for 3D Model Using Oblique Aerial Images. First International Conference on Intelligent Networks and Intelligent Systems (ICINIS 2008), pp. 495-498, Wuhan, China.

[10] L. Barazzetti, F. Remondino, M. Scaioni (2010). Orientation and 3D modelling from markerless terrestrial images: combining accuracy with automation. The Photogrammetric Record, (pp. 356- 381).

[12] R. Berry, G. Higgs, M. Langford, R. Fry, 2010. An evaluation of online gas-based landscape and visual impact assessment tools and their potentials for enhancing public participation in the Uk, WebMGS 2010, XXXVIII-4/W13

[11] Remondino, F., Rizzi, A., 2010: Reality-based 3D documentation of natural and cultural heritage sites - Techniques, problems and examples. Applied Geomatics, Vol.2(3): 85-100

[12] Cuca B., Brumana R., Scaioni M., Oreni D. [2011], "Spatial Data Management of Temporal Map Series for Cultural and Environmental Heritage", International Journal of Spatial Data Infrastructure Research (IJSDIR) Vol. 6 (2011).

[13] M. Santana Quintero, K. Van Balen, (2009) "Rapid and cost-effective assessment for world heritage nominations", 22nd CIPA Symposium, October 11-15, 2009, Kyoto, Japan

[14] European Landscape Convention, Council of Europe, Florence, 20.X.2000. European Spatial Development Perspective (ESDP) - Postdam, May 1999. http://ec.europa. eu/regional_policy/sources/docoffic/official/reports/som_en.etm

[15] Scottish Natural Heritage (2008). http://www.snh.org.uk/publications/online/ heritagemanagement/EIA/appendix1.shtml

[16] INSPIRE EU Directive (2007). Directive 2007/2/EC of the EU Parliament and of the Council (14 March 2007) establishing an Infrastructure for Spatial Information in the EU Community, Official Journal of the European Union, L 108/1(50), 25th April 2007. 
